Revista Arbitrada Interdisciplinaria KOINONIA

Año 2020. Vol V. N². Especial: Saber y Tecnología Popular Hecho el depósito de Ley: FA2016000010 ISSN: 2542-3088

FUNDACIÓN KOINONIA (F.K). Santa Ana de Coro. Venezuela.

Julissa Marlene Llanganate-Peñafiel; Luís Albarracín-Zambrano; Dionisio Vitalio Ponce-Ruíz

http://dx.doi.org/10.35381/r.k.v5i2.851

\title{
Sistema automático de alarma sísmica con Raspberry PI para el campus UNIANDES - Quevedo - Ecuador
}

\section{Automatic seismic alarm system with Raspberry PI for the UNIANDES campus - Quevedo - Ecuador}

\author{
Julissa Marlene Llanganate-Peñafiel \\ marlenellanganate@hotmail.com \\ Universidad Regional Autónoma de Los Andes, Quevedo \\ Ecuador \\ https://orcid.org/0000-0002-6642-5680 \\ Luís Albarracín-Zambrano \\ uq.luisalbarracin@uniandes.edu.ec \\ Universidad Regional Autónoma de Los Andes, Quevedo \\ Ecuador \\ https://orcid.org/0000-0002-3164-5229 \\ Dionisio Vitalio Ponce-Ruíz \\ manzanillo1962@gmail.com \\ Universidad Regional Autónoma de Los Andes, Quevedo \\ Ecuador \\ https://orcid.org/0000-0002-5712-4376
}

Recibido: 19 de mayo de 2020

Revisado: 10 de junio de 2020

Aprobado: 15 de julio de 2020

Publicado: 21 de julio de 2020 


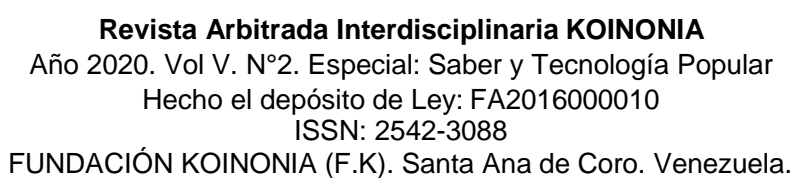

Julissa Marlene Llanganate-Peñafiel; Luís Albarracín-Zambrano; Dionisio Vitalio Ponce-Ruíz

\title{
RESUMEN
}

La sociedad del conocimiento tiene como uno de sus fundamentos el aprendizaje desde las TIC, lo cual implica no solo aprender un manejo instrumental, sino, crear, innovar, a través de los medios tecnológicos. La investigación tuvo por objetivo crear un sistema automático de alarma sísmica con Raspberry PI para el campus de la Universidad Regional Autónoma de Los Andes (UNIANDES) - Quevedo de Ecuador, empleando una metodología sistémica, para lo cual se procedió mediante cinco fases investigativas. Se diseñó y desarrolló el sistema automático, para así aplicar su funcionamiento en el campus de UNIANDES - Quevedo, generando así una rápida reacción al alertar un evento sísmico, teniendo en consideración los procedimientos y protocolos de seguridad adoptados por la institución, dedicando el estudio a las tecnologías y bondades que ofrece la tarjeta Raspberry pi 3 modelo B+ en la automatización de procesos.

Descriptores: Tecnología adecuada; desarrollo endógeno; cultura y desarrollo; sismómetro. (Palabras tomadas del Tesauro UNESCO).

\begin{abstract}
The knowledge society has as one of its foundations the learning from the ICT, which implies not only to learn an instrumental handling, but, to create, to innovate, through the technological means. The objective of the research was to create an automatic seismic alarm system with Raspberry PI for the campus of the Regional Autonomous University of Los Andes (UNIANDES) - Quevedo of Ecuador, using a systemic methodology, for which it proceeded through five investigative phases. The automatic system was designed and developed, in order to apply its operation on the UNIANDES - Quevedo campus, thus generating a quick reaction when alerting a seismic event, taking into account the security procedures and protocols adopted by the institution, dedicating the study to the technologies and benefits offered by the Raspberry pi 3 model $B+$ card in process automation.
\end{abstract}

Descriptors: Appropriate technology; endogenous development; Culture and development; seismometers. (Words taken from the UNESCO Thesaurus). 


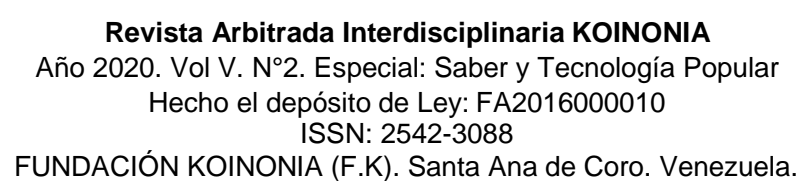

Julissa Marlene Llanganate-Peñafiel; Luís Albarracín-Zambrano; Dionisio Vitalio Ponce-Ruíz

\section{INTRODUCCION}

Ecuador se encuentra ubicado en el llamado cinturón de fuego, siendo un conjunto de territorios desde Asia hasta América, siendo esta zona considerada como la de mayor magnitud volcánica y sísmica, específicamente el Ecuador se encuentra inmerso en la placa de Nazca y placa Sudamericana, lo cual hace un país vulnerable a eventos sísmicos, siendo el último de gran magnitud ocurrido el 16 de abril de 2016:

El Terremoto de Pedernales tuvo una magnitud de momento Mw 7.8 y una intensidad máxima de IX (según la Escala macro sísmica europea, EMS-98) y se convirtió en el evento natural más catastrófico de lo que va del presente siglo y fue sentido en localidades tan distantes como Bogotá en Colombia y Cajamarca en Perú (Instituto Geofísico - EPN, 2020).

Lo descrito contribuye a tomar medidas preventivas para tratar de minimizar el impacto de los sismos que puedan ocurrir en el Ecuador, siendo pertinente configurar construcciones anti sísmicas, refugios, rutas de evacuación, alarmas sísmicas, siendo esto último, el punto central del actual trabajo investigativo, en este sentido, se hace necesario que la población se encuentre familiarizada con la actividad y riesgos sísmicos de la zona donde habitan, con la finalidad de actuar se genere la alarma, para lo cual es importante realizar ensayos previos a un posible evento sísmico, por cuanto esto elevará a eficacia del sistema de prevención (Tapia-Hernández, et al., 2017).

Desde la concepción de la macrobioética se toma responsabilidad social en contribuir en la preservación de la vida y del entorno ecológico (Álvarez-Díaz, 2020), lo cual contribuye a la conformación de una visión preventiva desde la proposición de un sistema de alarma sísmica, siendo esta opción importante de desarrollar por cuanto genera expectativas de articular una cultura preventiva en función de evitar males mayores a causa de sismos. Sin embargo, la falta de una cultura científica para el manejo acertado de las zonas de riesgo natural, así como del abordaje durante y después que ocurra un determinado fenómeno como un sismo, es una advertencia generada por (Alcántara-Ayala, et al., 2019), siendo indispensable promover desde las instituciones de educación superior, planes de educación pública en función de contribuir a un enfoque ciudadano en abordaje de la realidad social con compromiso bioético de defensa y promoción de la vida, siendo 


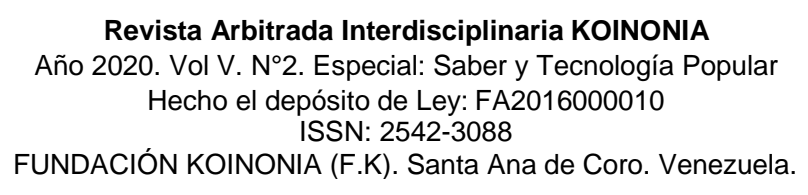

Julissa Marlene Llanganate-Peñafiel; Luís Albarracín-Zambrano; Dionisio Vitalio Ponce-Ruíz

una alternativa generar el desarrollo tecnológico con fines de solventar problemáticas cotidianas, en fusión de lo científico con el saber popular, así desde una concepción multidisciplinar se concibe el aporte a una cultura preventiva (Albarracín-Zambrano, et al., 2020).

Prosiguiendo lo descrito, los desarrollos tecnológicos desde las universidades deben ser diseñados en solventar problemáticas sociales en función de contribuir a la sostenibilidad del ambiente, optimización de recursos, contribuyendo en función de lo disponible, esto permite generar un aprendizaje autónomo centrado en el estudiante (Tartabull-Contreras, 2015).

Así se proyecta un aprendizaje activo en donde el estudiante diseña, crea, innova, en proposición de lograr progresivamente el crecimiento tecnológico tan necesario para complementar el progreso de los pueblos desde la ciencia, teniéndose como eje central la investigación para tal fin (Peche-Cruz \& Giraldo-Supo, 2019), así desde la innovación tecnológica se contribuye en fomentar el pensamiento lógico - matemático, estando en concordancia con lo planteado por la sociedad del conocimiento (Bermúdez-Tacunga, 2014).

La sociedad del conocimiento tiene como uno de sus fundamentos el aprendizaje desde las TIC, lo cual implica no solo aprender un manejo instrumental, sino, crear, innovar, a través de los medios tecnológicos, así la educación superior debe promover la adaptación de su currículo en función de posicionarse en los retos educativos actuales (GarcésSuárez, et al., 2016), así se contribuye además en trabajar en superar la brecha económica global, en función de ser la educación uno de los objetivos de desarrollo sostenible de cara al 2030 en cooperar en tal situación (Coppelli-Ortiz, 2018).

De ese modo, se tiene en cuenta el enfoque constructivista de aprendizaje, mediante el cual se genera conocimiento en la medida que el estudiante, construye el producto tecnológico en función de solventar una determinada debilidad o problemática, conjugándose teoría y práctica (Molina-Chalacan, et al., 2020), desde donde el pensamiento crítico - reflexivo se constituye en un eje fundamental para promover la innovación en razón del crecimiento social (Espinosa-Cristia, 2019). 


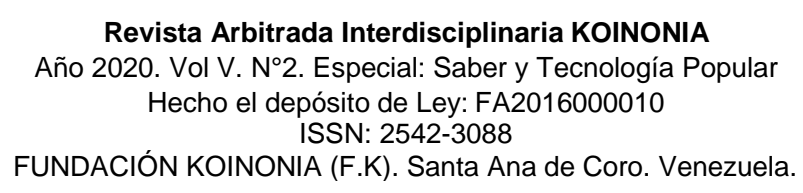

Julissa Marlene Llanganate-Peñafiel; Luís Albarracín-Zambrano; Dionisio Vitalio Ponce-Ruíz

En función de lo planteado, el siguiente producto tecnológico, tuvo por objetivo crear un sistema automático de alarma sísmica con Raspberry PI para el campus UNIANDES Quevedo de Ecuador, empleando una metodología sistémica, para lo cual se procedió del siguiente modo:

Fase I: Etapa de investigación indagatoria. Se recopiló la información acerca del protocolo a aplicar, tecnologías que existen en el mercado con pertinencia a las necesidades del proyecto.

Fase II: Análisis de tecnología. Se analizaron los tipos de tecnologías y los diferentes protocolos más oportunos para el desarrollo del sistema automatizado e implementación, se realizó pruebas de funcionalidad, así como también conocer las ventajas y desventajas de cada una, se ejecutó un estudio de los dispositivos como Raspberry Pi, sensor.

Fase III: Implementación del hardware. En esta fase tuvo como propósito principal, la adquisición de los dispositivos electrónicos a controlar, e implementación de los mismos, se desarrolló una arquitectura robótica que facilitó la creación del escenario según las necesidades expuestas, la arquitectura radicó en la posibilidad de integrar distintos dispositivos conectados al PC, finalmente se realizó la configuración de cada uno de los dispositivos y las pruebas de funcionalidad.

Fase IV: Diseño de la plataforma de aplicación. Durante esta fase se trabajó en el diseño de la plataforma de aplicación o sistema. El lenguaje de programación seleccionado para el desarrollo del sistema automatizado fue Python, alojado en la Raspberry Pi, la cual utiliza sistema operativo Linux (Suárez-López, et al., 2017).

Fase V: Montajes y pruebas. Se procedió a unir todas las etapas mencionadas y se realizaron las respectivas pruebas pertinentes para verificar la efectividad del prototipo diseñado. 


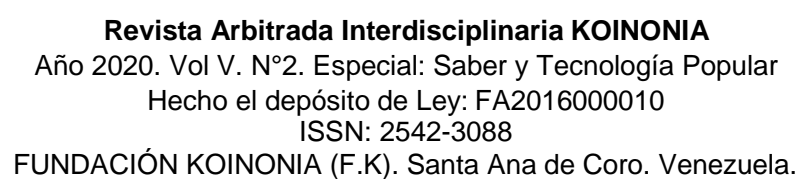

Julissa Marlene Llanganate-Peñafiel; Luís Albarracín-Zambrano; Dionisio Vitalio Ponce-Ruíz

\section{PROPUESTA TECNOLÓGICA}

La investigación de campo permitió recolectar la información necesaria, se encontró diferentes materiales de dispositivos eléctricos clasificados según sus propiedades, su funcionamiento y como contribuyen a la comunidad estudiantil. Con los materiales aptos, investigaciones y análisis de alarma de sensor sísmico, permitiendo un gran avance brindando una nueva comodidad a la sociedad, en este trabajo sintetiza como el sistema automático de alarma sísmica con Raspberry Pi, funciona y que hace cada uno de sus dispositivos integrados.

El sistema automático de alarma sísmica, tiene empleado varios dispositivos eléctricos, el funcionamiento de la alarma es que en el momento que existe un movimiento telúrico (leves-fuerte) se active, uno de sus principales componentes es un sensor de sensibilidad que permite sentir cualquier movimiento por más sensible, utilizando sus demás componentes como son los cables (jumper) cual nos ayuda a una conectividad mucho mejor, utilizando una sirena que en el momento de un movimiento se enciende y al mismo tiempo un foco de alarma para que las personas con discapacidad auditivas se guíen y de esta manera puedan entender lo que está sucediendo, todo integrado con una placa Raspberry Pi 3 modelo B+ el cual utiliza un sistema operativo Linux.

\section{Procedimiento}

Para la construcción del sistema se comenzó a conectar los componente electrónicos como la resistencia y zumbador o sirena (led en el mismo lugar) en cualquier parte del protoboard, luego se conectó en los pines de el mismo lado del zumbador dos cables jumpers, los cuales el negativo iría conectado a positivo del protoboard y el negativo del zumbador, el jumper va en el pin 10 de la Raspberry $\mathrm{Pi}$, en un lado de la resistencia colocamos un jumper y ese iría en pin del medio del sensor y atrás de ese jumper va otro que está conectado en positivo en el protoboard mientras tantos en la otro pin de la resistencia iría conectado en el protoboard en el negativo en nuestro sensor del primer pin estaría conectada en el positivo del protoboard, colocamos en nuestro Raspberry Pi en el GND un jumper que iría a positivo en el protoboard. 


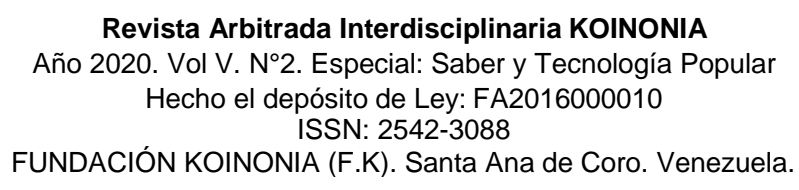

Julissa Marlene Llanganate-Peñafiel; Luís Albarracín-Zambrano; Dionisio Vitalio Ponce-Ruíz

También teniendo en cuenta una placa Raspberry $\mathrm{Pi}$, la cual utiliza un sistema operativo Linux donde se aloja la codificación para las órdenes de activar la alarma cuando el sensor perciba algún movimiento, de esta manera la comunidad universitaria podrá tomar las debidas prevenciones en caso de un sismo. Se hicieron varias pruebas en la comunidad universitaria donde se colocó la alarma sísmica en un lugar seguro, en los trascursos de los meses se desarrollaron sismos leves, se pudo observar que la alarma si estaba funcionando correctamente, porque la alarma se activó dando aviso a las comunidades universitarias que debían evacuar los edificios e ir a la zona segura. La alarma se mantuvo activa en el trascurso que duro el movimiento sísmico una vez que paso completamente la alama se silenció.

\section{Diagrama de flujo}

Se analizaron todos los sensores existentes en el mercado, seleccionando el de mayor nivel de sensibilidad indicado, seguido de esto el diseño del circuito para ver si los sensores y demás elementos electrónicos son actos si esto no funcionaban se volvía al análisis de sensores y dispositivos, caso contrario se comienza a elaborar la sistema automatizado, se realizaron las pruebas correspondientes, si estas tiene una falla se vuelve al paso anterior, caso contrario se realiza la implementación de la alarma sísmica y así llegando al fin de nuestro algoritmo, como se puede visualizar en la figura 1. 
Revista Arbitrada Interdisciplinaria KOINONIA

Año 2020. Vol V. N². Especial: Saber y Tecnología Popular Hecho el depósito de Ley: FA2016000010

ISSN: 2542-3088

FUNDACIÓN KOINONIA (F.K). Santa Ana de Coro. Venezuela.

Julissa Marlene Llanganate-Peñafiel; Luís Albarracín-Zambrano; Dionisio Vitalio Ponce-Ruíz
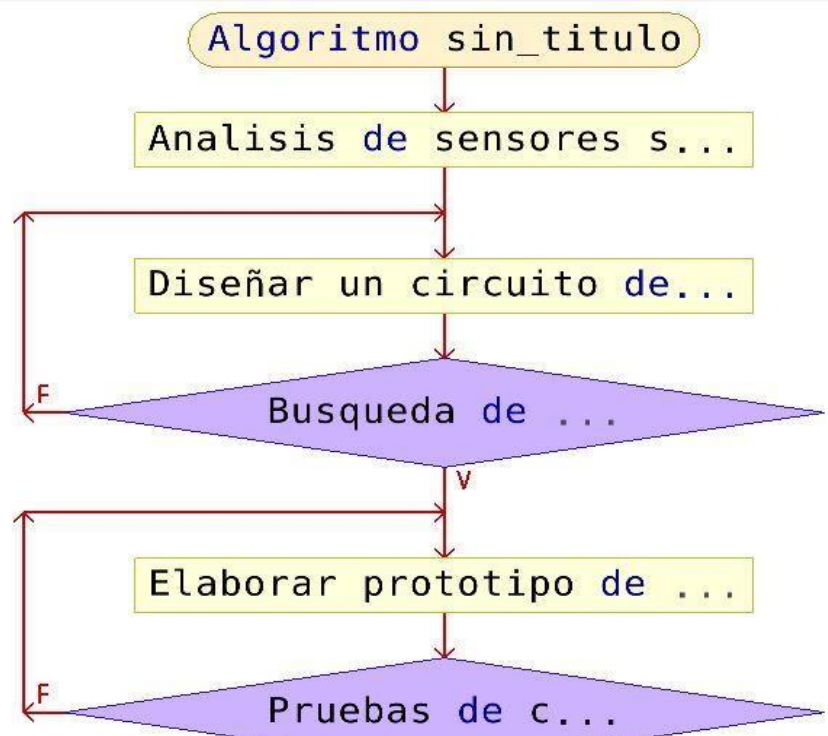

IV

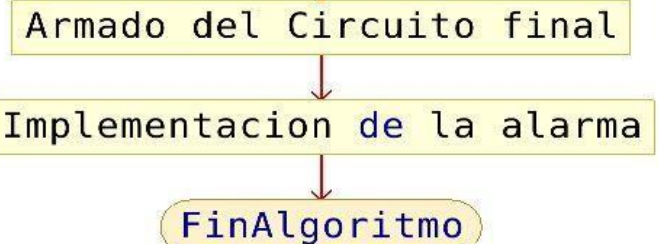

Figura 1. Diagrama de flujo (alarma con sensor). Fuente: Los autores. 
Revista Arbitrada Interdisciplinaria KOINONIA

Año 2020. Vol V. N². Especial: Saber y Tecnología Popular
Hecho el depósito de Ley: FA2016000010
ISSN: 2542-3088
FUNDACIÓN KOINONIA (F.K). Santa Ana de Coro. Venezuela.

Julissa Marlene Llanganate-Peñafiel; Luís Albarracín-Zambrano; Dionisio Vitalio Ponce-Ruíz

\section{Diagrama de bloque (alarma con sensor sísmico)}

Al momento de que el sensor de vibración sienta un movimiento este envía un input (entrada) a la Raspberry Pi este enviando un output (salida) alerta activando la sirena y led al mismo tiempo como se puede observar en la figura 2.

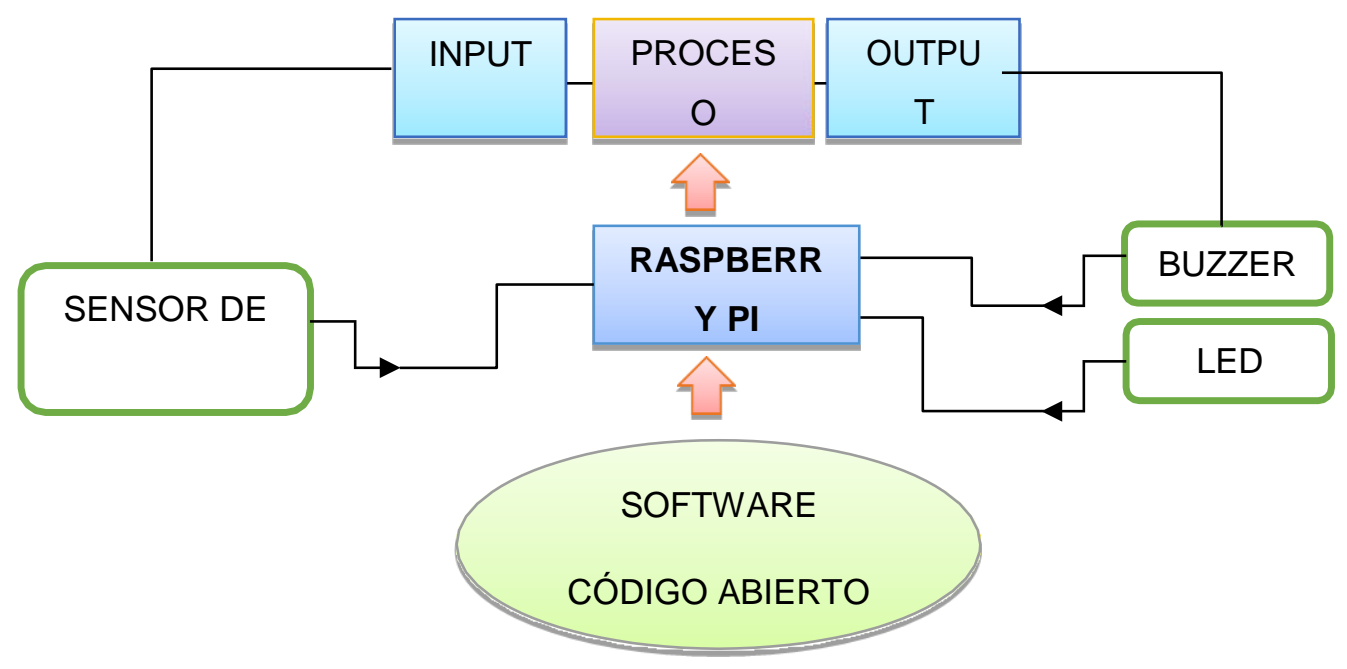

Figura 2. Diagrama de bloqueo. Fuente: Los autores 
Revista Arbitrada Interdisciplinaria KOINONIA

Año 2020. Vol V. No2. Especial: Saber y Tecnología Popular

Hecho el depósito de Ley: FA2016000010

ISSN: 2542-3088

FUNDACIÓN KOINONIA (F.K). Santa Ana de Coro. Venezuela.

Julissa Marlene Llanganate-Peñafiel; Luís Albarracín-Zambrano; Dionisio Vitalio Ponce-Ruíz

\section{Esquema grafico}

Se muestra las conexiones de nuestro circuito la ubicación de sus pines sus conexiones positivas y negativas para lograr el funcionamiento del sistema automatizado como se observa en la figura 3.

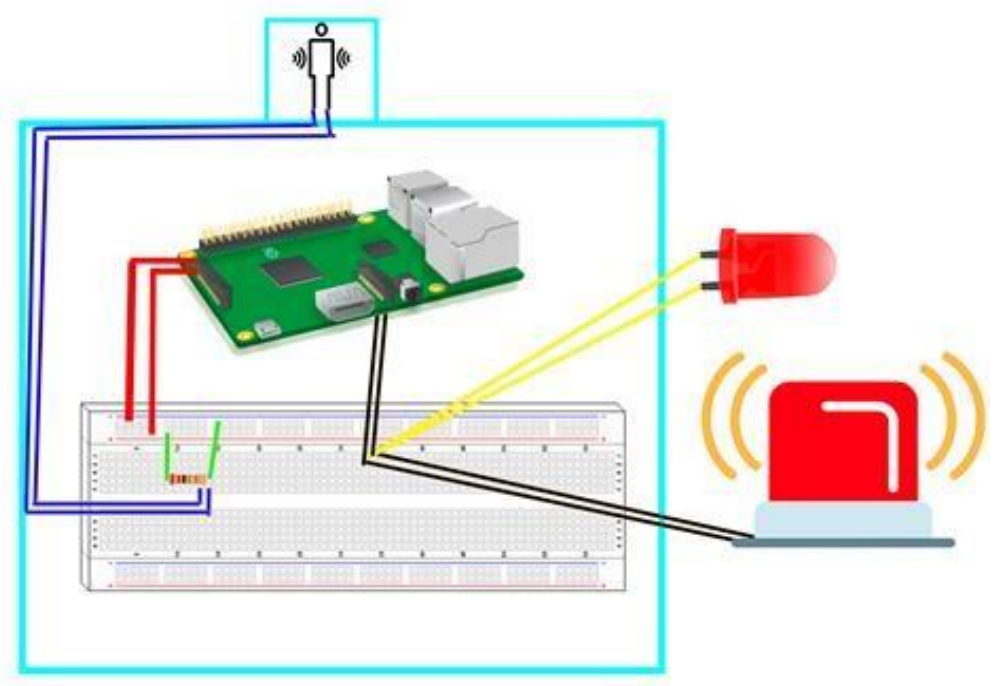

Figura 3: Esquema gráfico. Fuente: Los autores 
Revista Arbitrada Interdisciplinaria KOINONIA

Año 2020. Vol V. N². Especial: Saber y Tecnología Popular

Hecho el depósito de Ley: FA2016000010

ISSN: 2542-3088

FUNDACIÓN KOINONIA (F.K). Santa Ana de Coro. Venezuela.

Julissa Marlene Llanganate-Peñafiel; Luís Albarracín-Zambrano; Dionisio Vitalio Ponce-Ruíz

\section{Diseño final de la alarma de sensor sísmico}

En el diseño final se coloca el sistema automático de alarma sísmica en su caja de manera cuidadosa para proceder a su funcionamiento ya directo de su infraestructura como se observa en la figura 4 .

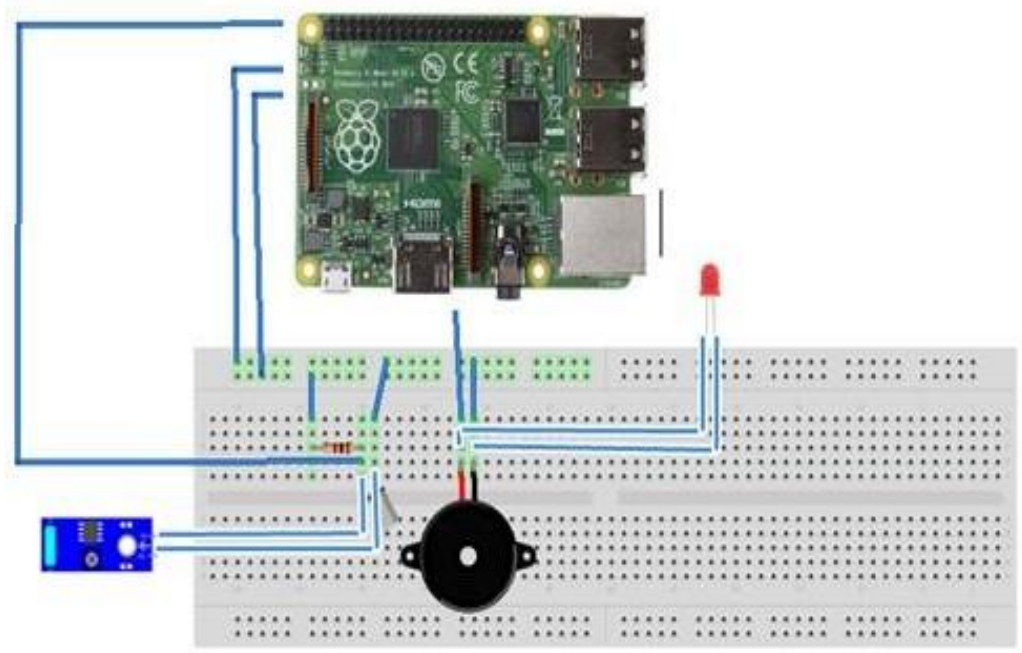

Figura 4. Diseño Final. Fuente: Los autores.

\section{CONCLUSION}

El desarrollo del sistema automático de alarma de sismos implementado en UNIANDES, Quevedo, ha permitido el desarrollo del pensamiento lógico creativo a través de un lenguaje de programación utilizando de dispositivos electrónicos y su aplicabilidad en la construcción equipos tecnológicos brindando una oportunidad de ser protagonistas en el aprendizaje. Se diseñó y desarrolló el sistema automático, para así aplicar su funcionamiento en el campus de UNIANDES - Quevedo, generando así una rápida reacción al alertar un evento sísmico, teniendo en consideración los procedimientos y protocolos de seguridad adoptados por la institución, dedicando el estudio a las 


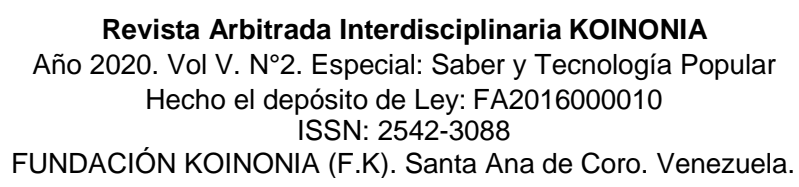

Julissa Marlene Llanganate-Peñafiel; Luís Albarracín-Zambrano; Dionisio Vitalio Ponce-Ruíz

tecnologías y bondades que ofrece la tarjeta Raspberry pi 3 modelo $B+$ en la automatización de procesos.

\section{FINANCIAMIENTO}

No monetario

\section{AGRADECIMIENTO}

A la Universidad Regional Autónoma de Los Andes, Campus Quevedo, por todo el apoyo brindado en la motivación y desarrollo de esta investigación.

\section{REFERENCIAS CONSULTADAS}

Albarracín-Zambrano, L. O., Jalón-Arias, E. J., Guerrero-Burgos, K. L., \& LlanganatePeñafiel, J. M. (2020). Prototipo de control de entrada y salida para el parqueadero de UNIANDES-Quevedo. [Entry and exit control prototype for the UNIANDESQuevedo parking lot]. Dilemas Contemporáneos: Educación, Política y Valores, 7(Especial febrero 2020), 1-10. https://doi.org/10.46377/dilemas.v33i1.2177

Alcántara-Ayala, I., Garza Salinas, M., López-García, A., Magaña-Rueda, V., OropezaOrozco, O., Puente-Aguilar, S., Rodríguez-Velázquez, D., Lucatello, S., RuizRivera, N., Tena-Núñez, R., Urzúa-Venegas, M., \& Vázquez-Rangel, G. (2019). Gestión Integral de Riesgo de Desastres en México: reflexiones, retos y propuestas de transformación de la política pública desde la academia. [Integrated Disaster Risk Management in Mexico: reflections, challenges, and proposals from the academic community seeking a transformation on policy making]. Investigaciones Geográficas, O(98). http://dx.doi.org/10.14350/rig.59784

Álvarez-Díaz, J. (2020). Género, desastres y mortalidad: Sismo en Ciudad de México, 19/septiembre/2017. [Gender, disasters and mortality: Earthquake in Mexico City, September 19th, 2017]. Ciência \& Saúde Coletiva, 25(7), 2831-2836. https://doi.org/10.1590/1413-81232020257.30802018

Bermúdez-Tacunga, R. (2014). El desarrollo tecnológico de la sociedad y sus incidencias en el pensamiento lógico matemático. Actualidades Investigativas en Educación, 14(2), 704-722. 


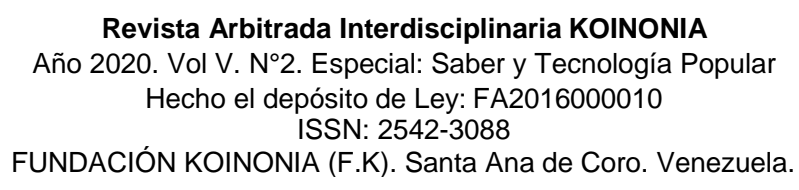

Julissa Marlene Llanganate-Peñafiel; Luís Albarracín-Zambrano; Dionisio Vitalio Ponce-Ruíz

Coppelli-Ortiz, G. (2018). La globalización económica del siglo XXI. Entre la mundialización y la desglobalización. [The economic globalization of the 21st Century. Between globalization and deglobalization]. Estudios internacionales (Santiago), 50(191), 57-80.

Espinosa-Cristia, J. (2019). Gestionando la innovación desde la óptica de los estudios de ciencia, tecnología y sociedad: por una perspectiva constructivista y crítica de la gestión de la innovación. [Managing innovation from the perspective of science, technology and society studies: from a constructivist and critical perspective of innovation management]. Cadernos EBAPE.BR, 17(1), 68-

83. https://doi.org/10.1590/1679-395171625

Garcés-Suárez, E, Garcés-Suárez, E, \& Alcívar-Fajardo, O. (2016). Las tecnologías de la información en el cambio de la educación superior en el siglo XXI: reflexiones para la práctica. [Information technologies in the 21st century change of higher education: reflections for practice]. Revista Universidad y Sociedad, 8(4), 171-177.

Instituto Geofísico - EPN (2020). Cuatro años después del terremoto de pedernales: un testimonio sobre el peligro sísmico en el Ecuador. [Four years after the flint earthquake: a testimony about the seismic hazard in Ecuador]. Recuperado de https://bit.ly/30yLmVA

Molina-Chalacan, L. J., Giler-Chango, J. L., \& Albarracín-Zambrano, L. O. (2020).

Prototipo para el control inmótico de oficinas UNIANDES Quevedo. [Prototype for imotic control of UNIANDES Quevedo offices]. Dilemas Contemporáneos: Educación, Política y Valores, 7(Especial febrero 2020), 1-10. https://doi.org/10.46377/dilemas.v33i1.2176

Peche-Cruz, H., \& Giraldo-Supo, V. (2019). El Aprendizaje Flip Learning centrado en el estudiante como generador de calidad educativa. [Student-centered Flip Learning as a generator of educational quality]. Revista Arbitrada Interdisciplinaria Koinonía, 4(8), 427-450. http://dx.doi.org/10.35381/r.k.v4i8.293

Suárez-López, D, Morales-Espinosa, R, Cordero-Gutiérrez, I, \& Schreiner-de-Oliveira, L. (2017). Diseño de una herramienta de medición de ruidos basados en tecnologías Arduino-Rasperry PI. [Design of a Noise Measure Tool Based on ArduinoRaspberry PI Technologies]. 12(1), 81-87. https://doi.org/10.22507/pml.v12n1a8

Tapia-Hernández, E, Reddy, E, \& Oros-Avilés, L. (2017). Retos e incertidumbres en la predicción y prevención del riesgo sísmico. [Challenges and uncertainties in the prediction and prevention of seismic risk]. Ingeniería sísmica, (96), 66-87. 
Revista Arbitrada Interdisciplinaria KOINONIA

Año 2020. Vol V. N². Especial: Saber y Tecnología Popular Hecho el depósito de Ley: FA2016000010

ISSN: 2542-3088

FUNDACIÓN KOINONIA (F.K). Santa Ana de Coro. Venezuela.

Julissa Marlene Llanganate-Peñafiel; Luís Albarracín-Zambrano; Dionisio Vitalio Ponce-Ruíz

Tartabull-Contreras, Y, Rivero-Casanova, C, \& Briones-Kusactay, V. (2015). El desarrollo tecnológico, la sostenibilidad, la gestión del conocimiento y el desarrollo social. [Technological development, sustainability, knowledge management and social development]. Revista Universidad y Sociedad, 7(3), 74-78.

2020 por los autores. Este artículo es de acceso abierto y distribuido según los términos y condiciones de la licencia Creative Commons Atribución-NoComercial-Compartirlgual 4.0 Internacional (CC BY-NC-SA 4.0) (https://creativecommons.org/licenses/by-nc-sa/4.0/). 